the length of the period of cannulation, particularly when this exceeded six hours. There should be a positive clinical indication if arterial cannulation is to be continued for longer than six hours, since the occurrence of overt ischaemic damage will probably be linked, albeit very rarely, to that of arterial occlusion. In the management of seriously ill patients, however, the slight chance of sustaining permanent ischaemic damage distal to the cannulation site must be weighed against the proved benefits of continuous monitoring of arterial blood pressure and ready access to arterial blood for respiratory gas analysis. The risks associated with arterial cannulation have yet to be compared with those of the multiple arterial punctures otherwise necessary to obtain information.
We thank our colleagues of the Nuffield Department of Anaesthetics for their co-operation and help in providing the basic information upon which this survey was based.

\section{References}

1 Zorab, J. S. M., Anaesthesia, 1969, 24, 431.

2 Mortenson, J. D., Circulation, 1967, 35, 118

${ }^{3}$ Bedford, R. F., and Wollman, H., Anesthesiology, 1973, 38, 228.

4 Allen, E. V., American fournal of Medical Science, 1929, 178, 237.

5 Jeresaty, R. M., American Heart fournal, 1968, 76, 481.

6 Brown, A. E., Sweeney, D. B., and Lumley, J., Anaesthesia, 1969, 24, 532.

7 Downs, J. B, et al., Anesthesiology, 1973, 38, 283.

${ }^{8}$ Bedford, R. F., Anesthesiology, 1975, 42, 219.

\title{
Pyogenic Cocci in Infantile Eczema throughout One Year
}

\author{
R. J. SMITH, V. G. ALDER, R. P. WARIN
}

disruptions of the child's life and normal activities. In many patients the condition is temporarily controlled by antibiotics, but in a few weeks or months a fresh phase of sepsis may occur. The multiple cracks and abrasions, the scaling and exudation, and the repeated trauma all favour secondary infection, but one problem is the origin of the organisms. Do they arrive on the skin from outside sources, or are they the same organisms which have remained in small numbers in some reservoir sites when the clinical infection has cleared?

To try to answer to this question we have studied a series of patients with infantile eczema and followed them up for one year. Regular swabs were taken and the organisms phage or serologically typed.

\section{Method}

The patients were taken from those with persistent childhood eczema (atopic dermatitis) attending the outpatient department at the Bristol Children's Hospital. They ranged in age from 16 months to 15 years, and all patients had eczema in at least four of the common sites, including the elbows, wrists, knee and ankle flexures, buttocks, buttock creases, hands and fingers, and the retroauricular area. We selected patients who would probably still have active eczema in a year's time because of its severity and chronicity, and who would be able to attend every few weeks for a year. Twenty-seven patients were admitted to the investigation, but seven failed to attend regularly for the whole year, and their results were discarded.

We used common treatments, including topical steroids. When it was thought to be clinically advisable a topical antibiotic-usually neomycin, gentamicin, or sodium fusidate-was also used. Also, short courses of an antibiotic by mouth were occasionally necessary for various infections, and penicillin, clindamycin, flucloxacillin, erythromycin, and oxytetracycline were given. We appreciated that the bacteria flora would to some extent be altered by these antibiotic treatments.

The patients were seen at various intervals from two to eight weeks, but usually every four weeks. Saline-moistened cotton wool swabs were taken from the nose, throat, and the most active eczema site. The clinical appearance of the eczema was noted, and a history of any septic lesion in the immediate family contacts was taken. Any family member accompanying the child had swabs taken from the nose, throat, and any lesion present. The parents were encouraged to bring other members of the family, but we could not screen the whole family in each case. The cotton wool swabs were plated within four hours on to nutrient blood agar medium and incubated aerobically and anaerobically for 24 hours.

The coagulase-positive staphylococci were then tested for their antibiotic sensitivities by means of a Multodisc (Oxoid), and phage typed by the method of Blair and Williams ${ }^{1}$ with the international basic set of phages. ${ }^{2}$
Departments of Dermatology and Pathology, Bristol Royal

R. J. SMITH, M.B., CH.B., Clinical Assistant in Dermatology

R. P. WARIN, M.D., F.R.C.P., Consultant Dermatologist 
The $\beta$-haemolytic streptococci were grouped serologically by Lancefield's method, and sent to the Streptococcal Reference Laboratory, Central Public Health Laboratory, Colindale, London, for confirmation and further serological typing of $M$ and $T$ antigens.

After a year a detailed chart was drawn up for each patient showing the clinical state, treatment, family infections, and the various bacteriological isolates during the whole period.

\section{Results}

It is not possible in a short paper to present each year's chart for all 20 subjects, and the results recorded pick out some of the salient points. Staphylococci and streptococci will be dealt with separately, though on many occasions both organisms were isolated.

\section{STAPHYLOCOCCI}

Incidence.-A coagulase-positive Staphylococcus aureus was isolated from the skin at some time in 18 of the 20 cases. In eight patients such bacteria were present on the skin for most of the period of observation, and in three they were present for about half the period. The staphylococci were more likely to be isolated from the skin when the eczema was severe, even when no obvious sepsis was present.

Phage Types.-The staphylococcal cultures isolated from the patients and members of their family gave phage patterns belonging to the phage groups shown in table I. During the investigation a patient may have been infected with one or more strains of staphylococci. The phage patterns occasionally varied within the groups, and sometimes a culture with a different phage group would emerge. Most of the variations occurred within phage group III and the mixed groups; in a few cases this occurred after the antibiotic treatment given to the patient (see below). Among the patients most of the cultures $(31.7 \%$ ) belonged to the mixed group (usually a combination of group III and group I phages, and occasionally with group II as well). Group III contained the next highest number of phage patterns $(21.9 \%)$. Together the two groups accounted for more than half $(53.7 \%)$ the cultures isolated. A similar distribution was found among the patients' families. If a patient yielded the same phage type of staphylococcus in several isolated swabs over some time we considered that he had carried for that period only one strain, which was allocated to one of the phage groups in the table. In seven cases the same phage type was isolated from the skin throughout most of the year. Ten patients had two or more attacks of clinical sepsis during the period of observation, and in five of these patients the same phage type of staphylococcus was present on the skin.

Nose Swabs.-Out of 196 nose swabs taken 90 were positive. In 11 of the 20 patients positive nose swabs coincided with positive skin swabs, and the same phage type was present on 55 out of 60 occasions when the swabs showed staphylococci from the skin and the nose at the same time.

Family Contacts.-Among the immediate family contacts a history of possible infected lesions was obtained in 12 patients. Similar histories were taken from matched controls among children attending outpatients with unrelated conditions such as warts, and in seven out of 20 cases there was a history of similar septic lesions in members of the family. This difference was not significant $(P>0 \cdot 2)$; nevertheless, in 14 out of 20 patients in the investigation the phage type of staphylococcus isolated from the family contacts was the same as that present on the oatient.
Antibiotic Sensitivity.-We did not intend to examine in detail the picture of varying antibiotic sensitivity of the staphylococci isolated. Many of the cultures were resistant to penicillin, and, particularly among the phage group III and mixed group, many were resistant to streptomycin, erythromycin, and neomycin. Nevertheless, certain points of interest arose. After topical treatment with neomycin nine patients who had previously had neomycin-sensitive staphylococci acquired neomycin-resistant staphylococci. This gave rise to nine cases when neomycin-resistant staphylococci were subsequently found among members of the families; in two of these cases the neomycin-resistant organism was present in a lesion (one septic finger, and one septic toe). After treatment with sodium fusidate five patients who had previously had staphylococci sensitive to sodium fusidate acquired staphylococci resistant to it. These too were subsequently found among members of the family. Neomycin or sodium fusidate resistance emerged only among the phage group III and mixed phage group strains and were associated with a modification of the original phage pattern. No resistance to gentamicin was noted. Further details of this aspect will be available in another paper.

\section{STREPTOCOCCI}

Incidence.-Haemolytic streptococci (Lancefield's group A(nine cases), group B (three cases), and other groups (one case)) were isolated from the skin in 13 out of 20 patients. In seven cases the bacteria were present on the skin more than once during the year, but in only three patients was the same serological type present. In subsequent attacks of sepsis streptococci were again isolated from the skin in only one patient, and even in this case a different serological type was present. When clinical sepsis was present streptococci were isolated on $16(27 \%)$ out of 60 occasions.

Serological Types. - The streptococci isolated are shown in table II. Group A type M 12 T 12 isolates are said to be associated with glomerulonephritis. Two other patients, who were excluded from this survey because they were observed for less than 12 months, were also infected with $\beta$-haemolytic streptococci group A type M $12 \mathrm{~T} 12$. The three patients from whom these streptococci were isolated showed no symptoms of nephritis, but another patient under our observation with secondary sepsis on infantile eczema from whom a group A type M 12 T 12 streptococcus was isolated developed a typical nephritis. Group $\mathrm{G}$ is common in dogs. One of the patients from whom this streptococcus was isolated had a close contact with a dog, but the significance of this was unknown.

Throat Swabs.-Throat swabs showed streptococci at some time in 10 out of the 20 patients. On 14 occasions positive throat swabs coincided with positive skin swabs, and in 12 of these the type was the same from both sites.

Family Contacts.-Throat swabs from the immediate family contacts showed streptococci in eight patients, and in seven of these the same serological type was present in the patient's skin lesion and the family contact throat swab. We could not estimate reliably histories of sore throats in family contacts.

\section{Discussion}

\section{STAPHYLOCOCCI}

The various systemic and topical antibiotics given may have affected the population of staphylococci in these cases and have

\begin{tabular}{|c|c|c|c|c|c|c|c|c|c|c|c|c|}
\hline No. of cultures isolated: & 1 & 1 & 3 & 1 & 4 & 3 & 1 & 1 & 1 & 1 & 2 & 2 \\
\hline $\begin{array}{l}\text { Lancefield's serological } \\
\text { group } \\
\text { Griffith type: } \\
\mathbf{M} \\
\mathbf{T}\end{array}$ & $\mathbf{A}$ & $\begin{array}{c}A \\
3 / 13 / B\end{array}$ & A & $\begin{array}{l}\text { A } \\
12 \\
12\end{array}$ & A & $\begin{array}{l}22 \\
12\end{array}$ & A & B type III & 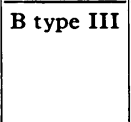 & $\bar{B}$ type III & C & $\mathbf{G}$ \\
\hline
\end{tabular}

TABLE I-Phage Groups of Staphylococci among 18 Patients and 17 Members of their Families

\begin{tabular}{|c|c|c|c|c|c|c|}
\hline Phage groups: & I & II & III & Mixed & Non-typable & Total \\
\hline $\begin{array}{l}\text { No. (\%) of staphylococci in phage groups: } \\
\text { Among patients } \\
\text { Among their families }\end{array}$ & $\begin{array}{l}7(17 \cdot 1) \\
6(18 \cdot 8)\end{array}$ & $\begin{array}{l}4(9 \cdot 8) \\
5(15 \cdot 6)\end{array}$ & $\begin{array}{l}9(21.9) \\
5(15 \cdot 6)\end{array}$ & $\begin{aligned} 13 & (31 \cdot 7) \\
8 & (25)\end{aligned}$ & $\begin{array}{l}8(19 \cdot 5) \\
8(25)\end{array}$ & $\begin{array}{l}41 \\
32\end{array}$ \\
\hline
\end{tabular}

TABLE II-Types of $\beta$-Haemolytic Streptococci Isolated 
encouraged different staphylococci, which may not have been sensitive. The antibiotic treatment, particularly the topical application, probably also changed the phage types. This subject will be discussed in a second paper. Nevertheless, in spite of the influence of these two factors the same phage type was isolated throughout most of the year in seven cases. Also, half the patients who had two or more attacks of clinical sepsis had the same phage type of staphylococci present in subsequent attacks. It seems therefore that the most likely source of the staphylococcus causing a second attack of clinical sepsis was a reservoir site on the patient, in which the same staphylococcus remained after the previous infection. Such reservoir sites were the eczematous areas or the nasal vestibule. The role of the nasal vestibule is emphasized by the fact that on 60 occasions nose and skin swabs both showed staphylococci, and in 55 of these the same phage type was present at both sites.

Though the incidence of a history of infective lesions in the family seemed high, control patients showed a similar incidence. Nevertheless, the fact that the same phage type of staphylococcus was often present in swabs from the family contacts suggests that such contacts may also be a source of the infecting staphylococcus.

\section{STREPTOCOCCI}

Streptococci were isolated less often than staphylococci, but, nevertheless, were present on some occasions in 13 out of 20 cases. In only seven cases were streptococci present more than once, and the same serological type of streptococcus was never found in subsequent attacks of clinical sepsis. Streptococci probably cause isolated attacks of secondary infection and are not likely to settle on the skin in a reservoir site. The association of streptococci of the same serological type in throat swabs of the patients and family contacts suggests that the streptococci come from sources other than the skin itself, and if streptococci are present in subsequent attacks then they too have reached the skin from outside sources and are usually of a different serological type.

\section{ASPECTS OF ANTIBIOTIC TREATMENT}

Though we do not intend to discuss treatment here the use of neomycin and sodium fusidate incorporated in steroid applications seems open to criticism. In the case of staphylococci these steroid applications might control some acute attacks of clinical sepsis when this organism is sensitive to the antibiotics, but with regard to preventing further attacks it seems that there is a ready acquisition of strains resistant to these drugs which is difficult to control. Furthermore, these resistant strains may spread to other family members. Streptococci are also highly resistant to neomycin and gentamicin and moderately resistant to sodium fusidate so that this type of clinical sepsis would also not be avoided by their use.

We thank Dr. M. T. Parker for the serological typing of the streptococci and for his advice. We also thank Professor W. A. Gillespie for his encouragement, and Dr. J. L. Burton for help with the presentation.

\section{References}

1 Blair, J. E., and Williams, R. E. O., Bulletin of the World Health Organization, 1961, 24, 771 .

${ }^{2}$ Subcommittee on Phage Typing of Staphylococci, International fournal of Systeinutic Bacteriology, 1971, 21, 167.

\title{
Plasma Corticotrophin Levels in Addison-Schilder's Disease
}

\author{
LESLEY H. REES， D. B. GRANT， J. WILSON
}

\section{Introduction}

Schilder's disease ${ }^{1}$ is characterized by non-inflammatory destruction of white matter but its exact nature is uncertain. In one form of the disease, a sex-linked condition, diffuse cerebral sclerosis is associated with adrenocortical insufficiency (AddisonSchilder's disease). ${ }^{2}$ Steroid replacement does not modify the fatal neurological course, and the response to exogenous corticotrophin (ACTH) may be impaired or absent. We are unaware of any data on circulating endogenous ACTH levels in such patients and report here the results of plasma ACTH assays in five boys with the disorder and in a symptom-free brother of one of them.

\section{Patients and Methods}

The disorder was characterized by dementia, cortical blindness or deafness, pyramidal signs, and ataxia. The clinical features (see table I) are detailed elsewhere. ${ }^{3}$ Two boys (cases 1 and 2 ) had abnormal skin pigmentation. None had received steroids.

Urinary 17-hydroxycorticoids (17-OHCS) and 17-oxosteroids were measured by Cathro's ${ }^{4}$ methods, and plasma cortisol by competitive protein binding before and 30 minutes after an intravenous injection of $0.25 \mathrm{mg}$ tetracosactrin. ${ }^{5}$ Plasma ACTH was estimated in samples collected into heparinized plastic tubes, enzymatic degradation being prevented. ${ }^{6}$ Two antisera were used for the radioimmunoassay, one against the active $\mathrm{N}$-terminal part of the ACTH molecule and the other against the inactive $\mathrm{C}$ terminus. ${ }^{7}$ Measurements were also made 